\title{
Babywearing practice and unnoticed danger
}

\section{INTRODUCTION}

Babywearing is the practice of carrying a baby that ensures an ongoing contact between the baby (in a vertical position) and the adult, allowing the wearer to have two free hands. There are many different baby carriers: wraps, ring slings, softstructured (or buckle) carriers, and backpacks.

Babywearing became exponentially popular thanks to social media. Parents with a common interest in this topic got together, and programs aimed at training babywearing coaches emerged. This practice, promoted as part of a mother-child dyad bonding process, may turn into a major tragedy as a result of positional asphyxia or milk aspiration if the baby is breastfeeding during babywearing. ${ }^{1}$

The United States Consumer Product Safety Commission (CPSC) reported a total of 14 deaths attributed to sling carriers in a 20-year period (1990-2010), 12 of them in infants younger than 4 months. ${ }^{2}$ More recently, a European publication reported 19 cases over a 6-year period in France and Belgium, most also in infants younger than 4 months. $^{3}$

\section{Vulnerabilities of baby carriers}

Relative macrocephaly in newborn infants and hypotonia typical of preterm newborn infants and underweight children puts them in danger. The risk of babywearing encompasses three potential positional asphyxia mechanisms: 1) crushing of the baby's nose and mouth against the wearer's body; 2) head flexion against the trunk, leaving the jaw in a posterior position and the tongue touching the soft palate, thus causing air obstruction in the posterior pharynx, ${ }_{i}^{4} 3$ ) risk for asphyxia due to having the face against the cloth. These three mechanisms may be worse when the size of the upper airways is inadequate, as in the case of children with micrognathia, which is often familial.

Breastfeeding is the riskiest activity associated with babywearing because the baby remains at a lower height and the airways are not so easily visible, so they may suffer positional asphyxia, choking, or milk aspiration. Children with a cold may have intermittent hypoxia and/or obstructive apnea. ${ }^{5}$ Such hypoxic scenario may contribute to slow physiological protective mechanisms, i.e., awakening and autoresuscitation. ${ }^{6}$
In addition, children with respiratory conditions may have trouble falling asleep ${ }^{7}$ and tend to catch up while being carried in these devices. Such catch-up sleep is deep and requires a higher stimulus threshold for arousal.

\section{Vulnerabilities of early verticalization}

Newborn babies and / or young infants cannot hold their head in a vertical position and tend to neck hyperextension and / or hyperflexion. Such head swaying is perceived as a loss of balance and visual plane. As a defense, the baby raises the shoulders and presses the arms against the body to fix the head; this is done at the expense of hypertonia, which translates into discomfort and irritability. In the end, it implies over-adaptation because the maturity required to hold the head has not developed yet. ${ }^{8}$

Verticality defies gravity. Humans found a way of adapting until walking on two legs by developing synergistic mechanisms of muscle and tone coordination to ensure such erectness, and such synergy evolved over dozens of thousands of years. This is a process whereby humans distanced from the bearing plane, keeping the necessary distance between the vertebrae and controlling balance both during rest and movement. These synergies develop through the subject's own experience and facing gravity.

In vertical position, the infant's back constitutes a large kyphotic curve. The baby is clearly not ready to sit on their own but has been seated by someone else. This is because the muscle synergies that hold the spine have not yet been formed: rectus abdominis muscle, ischialfibular-tibial bundle, gluteus muscle, lumbar muscle, iliopsoas muscle.

Once standing on two legs is achieved, the anterior-posterior curves of the spine that make up for the weight of the different body segments have developed: cervical and lumbar lordosis, thoracic and sacrococcygeal kyphosis. Muscle tone adaptation to gravity, which throughout development depends on labyrinthine sensitivity, changes along by means of global dexterity, considering all body parts at the same time. Placing the baby in a position that their body has not yet achieved on its own implies depriving them from such natural synergistic coordination mechanism. ${ }^{9}$ 


\section{Attachment or clinging}

Babies do not need to be constantly in contact with the adult's body. Attachment accounts for a deep and very personal bond between the baby and their mother or adult caregiver, thus allowing the baby to meet their needs, ease their anxiety, and feel emotionally safe. Attachment develops especially during personal care moments, a privileged time of communication between the dyad.

Constant contact would prevent the baby from moving freely and unfolding their initiatives. Attachment and clinging are different situations. In addition, throughout development, there is a contradiction between attachment and exploration, with a safe emotional basis that allows a successful exploration. While the adult creates favorable conditions (safe space, relevant objects, positional safety), the child progressively discovers the world and formulates hypotheses about its rules. During these moments, there is no need for a continuous adult presence; their role as safeguard from a certain distance is enough. ${ }^{10}$

\section{Based on currently available evidence, the following is recommended:}

- Safety car seats, strollers, and the different types of baby carriers are not recommended for the baby to sleep in at home.

- The baby should be placed in the baby carrier face-up, with their back supported, and trying to prevent the face from crushing against the wearer's body.

- The face should always be uncovered.

- Do not feed the baby while carrying them in the device and consider the post-feeding interval.

\section{CONCLUSIONS}

If this type of device is selected to carry a baby, parents should be warned about the potential dangers in infants younger than 4 months and children with risk factors for sudden infant death syndrome (SIDS), such as prematurity, low birth weight, intercurrent respiratory disease. In terms of safety and for the purpose of favoring a healthy development, the most adequate device should meet the following requirements: the baby's back should be supported horizontally and their face should be visible so that the wearer can check that the nose and mouth are uncovered. Carrying the baby using a wide wrap folded in a triangle and placed as a sling appears to respect the physiological position and prevent the risk for asphyxia.

Alejandro Jenik, M.D.

Physician at the Department of Pediatrics and Neonatology of Hospital Italiano de Buenos Aires,

Autonomous City of Buenos Aires jenik@hospitalitaliano.org.ar

Estela Grad, M.D.

Task force on Sleep Disorders and Sudden Unexpected Infant Death Committee on Social Pediatrics Sociedad Argentina de Pediatría.

http: / / dx.doi.org/10.5546/ aap.2021.eng.8

To cite: Jenik A, Grad E. Babywearing practice and unnoticed danger Arch Argent Pediatr 2021;119(1):8-9.

\section{REFERENCES}

1. Batra EK, Midgett JD, Moon RY. Hazards Associated with Sitting and Carrying Devices for Children Two Years and Younger. J Pediatr. 2015;167(1):183-7.

2. Consumer ProductSafety Commission(USA). Infant deaths prompt CPSC warning about sling carriers for babies. [Accessed on:October 8 ${ }^{\text {th }}$, 2020]. Available at: https: / / www. cpsc.gov/ content/infant-deaths-prompt-cpsc-warningabout-sling-carriers-for-babies.

3. Bergounioux J, Madre C, Crucis-Armengaux A, BriandHuchet E, et al. Sudden deaths in adult-worn baby carriers: 19 cases. Eur J Pediatr. 2015;174(12):1665-70.

4. Tonkin SL, Vogel S, Bennet L, Gunn AJ. Positional upper airways narrowing and an apparent life threatening event. N Z Med J. 2002;115(1152):193-4.

5. Serebrovskaya T, Xi L. Intermittent hypoxia in childhood: the harmful consequences versus potential benefits of therapeutic uses. Front Pediatr. 2015;3:44.

6. Ramirez JM, Ramirez SC, Anderson TM. Sudden Infant Death Syndrome, Sleep, and the Physiology and Pathophysiology of the Respiratory Network. In Duncan JR, Byard RW (Eds.). SIDS Sudden Infant and Early Childhood Death: The Past, the Present and the Future. Adelaide (AU): University of Adelaide Press; 2018. [Accessed on: October $\left.8^{\text {th }}, 2020\right]$. Available at: https: / / www.ncbi.nlm. nih.gov/books/NBK513387/.

7. Simpson JM. Infant stress and sleep deprivation as an aetiological basis for the sudden infant death syndrome. Early Hum Dev. 200;61(1):1-43.

8. Falk J. Mirar al niño. La Escala de Desarrollo del Instituto Pikler (Lóczy). Buenos Aires: Ariana; 1997.

9. Szanto Feder A. Una mirada adulta sobre el niño en acción: el sentido del movimiento en la protoinfancia. Buenos Aires: Ediciones Cinco; 2014.

10. Chokler MH. Los organizadores del desarrollo psicomotor. Buenos Aires: Paidós; 1997. 